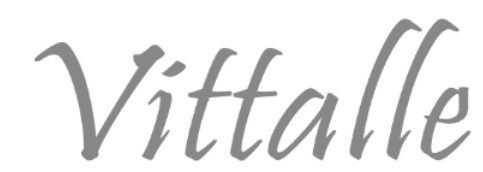

\title{
Terapia assistida por cães na pediatria oncológica: uma revisão integrativa
}

\author{
Amanda Gabrielly de Alcântara Paes*, Ana Clara Sueno Toda, Ana Júlia Mendes \\ Simão, João Victor Ferraiolli Gabrelon, Juliana Garnica Naufal, Maria Luísa Quadros \\ Giovanetti, Maria Elisa Gonzalez Manso \\ Centro Universitário São Camilo, São Paulo, SP, Brasil
}

\author{
Histórico do Artigo \\ Recebido em: \\ 24/01/2021 \\ Aceito em: \\ 01/07/2021
}

Palavras-chave:

Terapia assistida; cães; pediatria oncológica

Keywords:

Assisted therapy; dogs; pediatric oncology

\begin{abstract}
RESUMO
A Terapia Assistida por Animais (TAA) consiste em um método terapêutico que utiliza o animal escolhido como recurso no tratamento, podendo ser um facilitador entre o profissional da saúde e o paciente. Dentre os benefícios que essa terapia apresenta, destaca-se a maior socialização que o animal promove, a amenização de sintomas depressivos e a promoção de um ambiente mais descontraído. Diante do exposto, o artigo visa analisar os benefícios da terapia assistida por cães como tratamento coadjuvante na pediatria oncológica, visto que crianças submetidas ao tratamento quimioterápico podem apresentar prejuízos psicológicos, motores e sociais. Foi realizada uma revisão Integrativa nas bases PubMed, BVS e CAPES, no período entre 2015 e 2020, utilizando Descritores em Ciências e Saúde (terapia assistida, cães, pediatria oncológica) e Medical Subject Headings (dog assisted therapy, oncology pediatric, dogs, pet therapy). Seis artigos foram incluídos na síntese. Crianças submetidas a terapia assistida por cães ficaram mais alegres, ativas, mais comunicativas e também apresentaram diminuição do estresse. Em suma, apesar de se tratar de um estudo pouco explorado, diversos benefícios foram comprovados para o paciente. É um facilitador do trabalho médico, provando-se como um grande aliado à terapia convencional e promovendo uma maior adesão ao tratamento.
\end{abstract}

Dog-assisted therapy in pediatric oncology: an integrative review

\begin{abstract}
Animal-assisted therapy (AAT) is a therapeutic method that uses the chosen animal as a resource in the treatment and can be a facilitator between the health professional and the patient. Among the benefits which this therapy presents, is the greater socialization that the animal promotes, the relieve of depressive symptoms, and the promotion of a more relaxed environment. Therefore, the article aims to analysis of benefits of dog assisted therapy as a supporting treatment in pediatric oncology, since children submitted to chemotherapy treatment can present psychological, motor and social damages. An Integrative review was conducted on PubMed, BVS and CAPES bases in the period between 2015 and 2020 using Descritores em Ciência e Saúde (terapia assistida, cães, pediatria oncológica) and Medical Subject Headings (dog assisted therapy, oncology pediatric, dogs, pet therapy). Six articles were included in the synthesis. Children submitted to dog assisted therapy became happier, more active, more communicative, and also showed decrease of stress. In short, despite having been little explored, such study is proved to have generated several benefits for the patient, and it still acts as a facilitator in medical work, seen as a great ally to conventional therapy while promoting greater adherence to treatment.
\end{abstract}

\section{Introdução}

A relação homem-animal encontra-se presente na vida humana desde a antiguidade, com destaque para os cães, os quais contribuíram para a realização de atividades essenciais, por auxiliarem na caça e agricultura. Esses fatores foram decisivos para a aproximação e o estreitamento da relação homem-animal, propiciando a domesticação.

\footnotetext{
* Autor correspondente: amanda.paes@aluno.saocamilo-sp.br (Paes A.G.A.)
} 
Uma provável explicação para esta aproximação baseia-se na hipótese de Biofilia, proposta por Edward Osborne Wilson, em 1984, que destaca a necessidade biológica humana de se relacionar com a natureza e construir vínculos emocionais com outras formas de vida, principalmente os animais, o que promoveria bem-estar psicossocial (1).

$\mathrm{O}$ crescente reconhecimento dos benefícios da interação humano-animal resultou na utilização de animais com a finalidade terapêutica ou terapia assistida por animais (TAA), realizada, principalmente, com idosos e crianças portadoras de distúrbios cognitivos ou emocionais, tais como Autismo, Síndrome de Down e Quadros demenciais. Além disso, a TAA pode ser utilizada ainda como coadjuvante em casos de terapêutica de cânceres com antineoplásicos, com o objetivo de melhorar o bem-estar e as condições de saúde do paciente.

Apesar da TAA, segundo registros, ter sido utilizada no século IX, na Bélgica com pássaros, foi na metade do século XX que um psicólogo infantil de Nova York, chamado Boris Levinson, introduziu os cães no tratamento terapêutico, tendo realizado diversos experimentos e se consagrado como um dos maiores estudiosos do tema. Estes estudos demonstraram que os pacientes, na presença do cão, conseguiam demonstrar mais suas emoções e reduzir a quantidade de remédios utilizados (2).

A TAA utilizando cães ou Cinoterapia tem como base a sensibilidade, concentração e socialização, podendo ser uma ferramenta de apoio fundamental para o tratamento de crianças diagnosticadas com câncer (3).

A escolha do cão se faz pela facilidade de adestramento, pelo fato de apresentarem uma afeição natural pelas pessoas e, principalmente, pela capacidade de criar respostas positivas ao toque, uma vez que estímulos sensitivos, como o tátil, auditivo e visual, desencadeiam uma sensação prazerosa nos animais, os quais correspondem com gestos afetivos. Além disso, o cão pode contribuir para uma maior socialização das crianças (4).

Estudos relatam que a TAA promove redução da ansiedade, do estresse, da tristeza e da depressão. Além disso, a interação com os animais também favorece o aumento da atividade física realizada pelo paciente, melhora a relação entre a equipe e o paciente e incentiva as relações com novas pessoas (5).

A presente revisão objetivou verificar o impacto da TAA com cães na oncopediatria, de modo a apresentar a importância de um tratamento coadjuvante na saúde e bem-estar do paciente.

\section{Metodologia}

Trata-se de revisão integrativa, efetuada no segundo semestre de 2020, que buscou responder a seguinte pergunta norteadora: "Quais os benefícios da terapia assistida por cães como tratamento coadjuvante na oncopediatria?". Para tanto foram utilizados artigos originais encontrados nas bases de dados PubMed, BVS e CAPES, compreendendo o período de 2015 a 2020.

Os DeCS utilizados foram: terapia assistida, cães, pediatria oncológica e suas respectivas versões em inglês como termos MeSH. A combinação de descritores foi realizada por meio do operador booleano $A N D$.

Adotaram-se como critérios de inclusão: a) artigos publicados período entre 2015 e outubro de 2020; b) pesquisas relacionadas a terapia assistida por cães cujo enfoque se dava unicamente no tratamento coadjuvante na oncopediatria; c) idioma em português, inglês e espanhol; d) artigos completos (pagos e gratuitos); e) ensaios clínicos, estudos de prevalência, estudos de corte e de caso controle; f) pesquisas qualitativas.

Excluíram-se: a) estudos anteriores ao ano de 2015; b) artigos que não abordaram os critérios de inclusão acima; c) estudos com o foco apenas no animal; d) estudos com 
outros animais que não cães; e) estudos com modelos experimentais animais; f) séries de casos, relatos de caso, revisões não sistemáticas e opiniões de especialistas.

Foram identificados 27 artigos nas bases acima explicitadas. Os artigos passaram então por processo de verificação e análise, conforme apresentado na Figura 1, baseada no protocolo PRISMA (Principais Itens para Relatar Revisões Sistemáticas e Meta-análises), checklist com 27 itens e um fluxograma de quatro etapas (6). Os artigos passaram por um processo inicial de verificação e triagem, primeiramente através da leitura e a análise dos títulos e resumos, excluindo-se artigos que não atenderam à proposta deste estudo. Posteriormente, foi realizado o cruzamento entre as bases para retirar os duplicados, restando 14 artigos. Estes passos foram realizados em programa Word- Microsoft Office 365®. A seguir os 14 artigos selecionados foram novamente confrontados com os critérios de inclusão e exclusão, permanecendo apenas seis artigos para comporem esta revisão, os quais passaram a ser avaliados por todos os autores desta revisão, não havendo discordâncias quanto à sua inclusão.

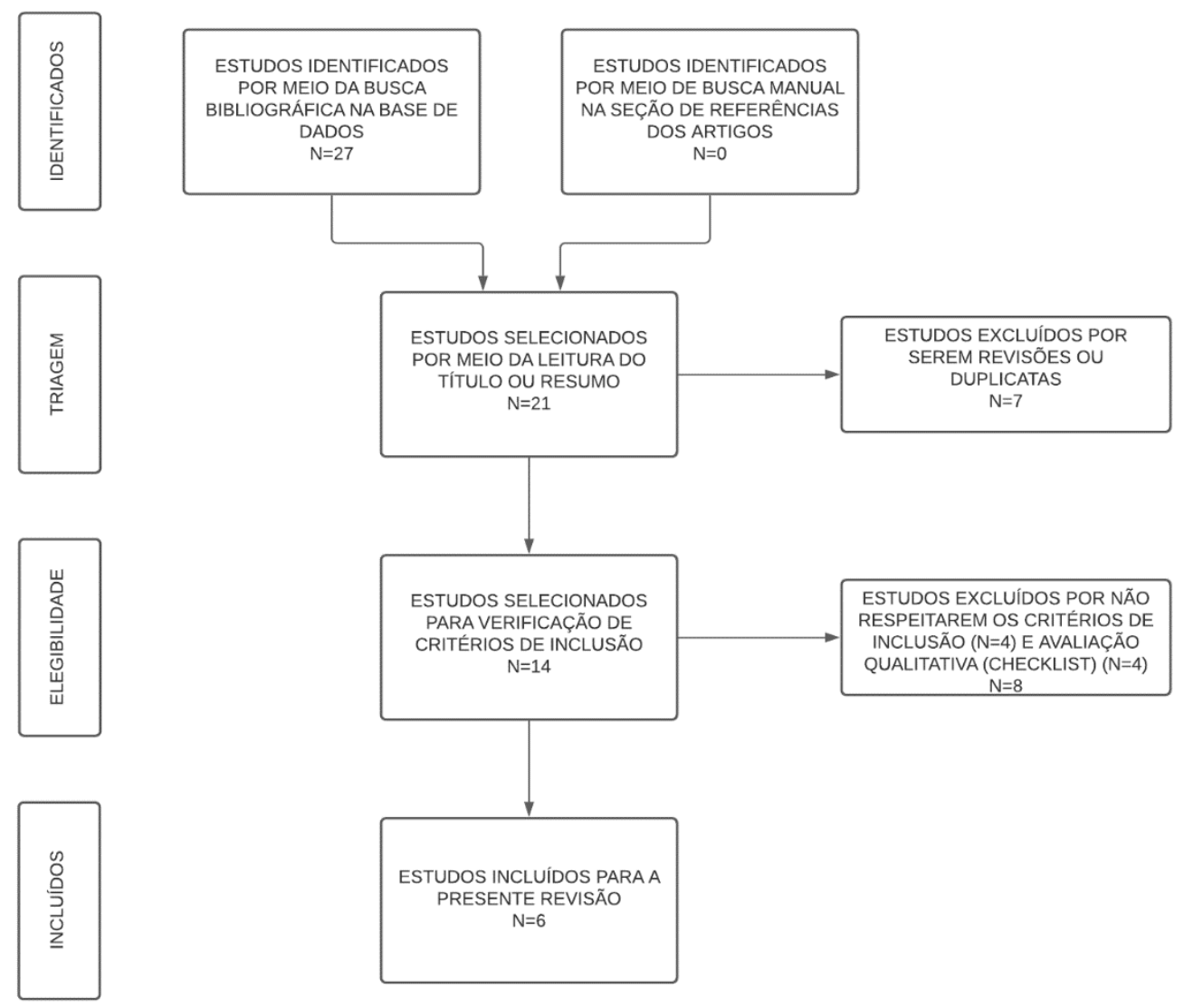

Figura 1 - Fluxograma de artigos incluídos na revisão sistemática, baseado em Moher et al. (6).

\section{Resultados}

Os artigos selecionados encontram-se sumarizados no Quadro 1 e organizados quanto ao autor e ano, amostra, objetivo, método e resultados. 
Quadro 1 - Artigos obtidos pela revisão, entre os anos de 2015 e 2020, com a temática dos descritores: Terapia assistida; Cães; Pediatria oncológica

\begin{tabular}{|c|c|c|c|c|}
\hline Autor e Ano & Amostra & Objetivo & Método & Resultados \\
\hline $\begin{array}{c}\text { Anne Ingalls } \\
\text { Gillespie e } \\
\text { Madalynn Neu } \\
2020 \\
(25)\end{array}$ & $\begin{array}{c}15 \text { crianças e } \\
\text { adolescentes de } 7 \text { a } 16 \\
\text { anos de idade, sendo } 7 \\
\text { meninos e } 8 \text { meninas. } 14 \\
\text { pacientes são } \\
\text { caucasianos e um } \\
\text { hispânico. }\end{array}$ & $\begin{array}{c}\text { Explorar as } \\
\text { experiências dos } \\
\text { participantes do } \\
\text { Youth and Pet } \\
\text { Survivors ao longo } \\
\text { do tempo e explorar } \\
\text { como a terapia } \\
\text { assistida por animais, } \\
\text { no meio virtual, pode } \\
\text { ser uma intervenção } \\
\text { adicional ou } \\
\text { alternativa à forma } \\
\text { tradicional da terapia } \\
\text { assistida por animais, } \\
\text { a qual envolve } \\
\text { visitas, ao vivo, com } \\
\text { cães principalmente. }\end{array}$ & $\begin{array}{c}\text { Foram feitas } \\
\text { entrevistas abertas e } \\
\text { gravadas digitalmente } \\
\text { com os pacientes, } \\
\text { durante o envolvimento } \\
\text { com os respectivos } \\
\text { animais usados na } \\
\text { terapia. }\end{array}$ & $\begin{array}{c}15 \text { crianças e } \\
\text { adolescentes que } \\
\text { participaram da pesquisa, } \\
\text { desenvolveram uma } \\
\text { conexão e amizade com o } \\
\text { animal. Além disso, a } \\
\text { escrita de cartas, na } \\
\text { terapia, se mostrou como } \\
\text { uma forma de dividir a } \\
\text { experiência do câncer } \\
\text { com um cachorro ou } \\
\text { gato, o qual também tem } \\
\text { câncer ou outra doença } \\
\text { grave. }\end{array}$ \\
\hline $\begin{array}{c}\text { Jessica Chubak e } \\
\text { Rene Hawkes } \\
2017 \\
(21)\end{array}$ & $\begin{array}{c}\text { Pacientes internados } \\
\text { com a idade de } 7 \text { a } 25 \\
\text { anos na unidade de } \\
\text { oncologia que estiveram } \\
\text { presentes em pelo menos } \\
1 \text { dos } 25 \text { potenciais de } \\
\text { consultas com cães de } \\
\text { terapia eram elegíveis } \\
\text { para este estudo. }\end{array}$ & $\begin{array}{l}\text { Este estudo avaliou a } \\
\text { viabilidade de estudar } \\
\text { atividades assistidas } \\
\text { por animais em } \\
\text { oncologia pediátricas } \\
\text { com internação e } \\
\text { coletou dados } \\
\text { preliminares sobre os } \\
\text { benefícios potenciais } \\
\text { do AAA para esta } \\
\text { população. }\end{array}$ & $\begin{array}{l}\text { A intervenção do estudo } \\
\text { consistiu em uma visita } \\
\text { única entre uma equipe } \\
\text { de cães de terapia e cada } \\
\text { paciente. A visita } \\
\text { ocorreu no quarto do } \\
\text { hospital privado de cada } \\
\text { paciente. A equipe do } \\
\text { cão de terapia foi } \\
\text { instruída a conduzir sua } \\
\text { visita. As visitas foram } \\
\text { limitadas a } \\
\text { aproximadamente } 20 \\
\text { minutos. } \\
\text { Implementamos } \\
\text { essa restrição de tempo } \\
\text { para permitir várias } \\
\text { visitas por dia. }\end{array}$ & $\begin{array}{l}\text { Após a visita do cão de } \\
\text { terapia, os pacientes } \\
\text { tiveram menos } \\
\text { sofrimento e diminuições } \\
\text { significativas na } \\
\text { preocupação, cansaço, } \\
\text { medo, tristeza e dor. }\end{array}$ \\
\hline $\begin{array}{c}\text { Macy Burr e Peggy } \\
\text { Wittman } \\
2020 \\
\text { (11) }\end{array}$ & $\begin{array}{l}\text { As entrevistas foram } \\
\text { feitas com dois } \\
\text { terapeutas, dois pais, um } \\
\text { voluntário, uma } \\
\text { recepcionista/auxiliar } \\
\text { administrativa e uma } \\
\text { aluna de Terapia } \\
\text { Ocupacional, além da } \\
\text { observação de pacientes } \\
\text { pediátricos durante três } \\
\text { semanas, ademais } \\
\text { incluíram momentos em } \\
\text { que o cão da terapia } \\
\text { estava presente e quando } \\
\text { não estava para verificar } \\
\text { a melhora social do } \\
\text { ambiente e dos pacientes } \\
\text { pediátricos. }\end{array}$ & $\begin{array}{l}\text { Demonstrar como o } \\
\text { tratamento com cães } \\
\text { pode melhorar a } \\
\text { motivação, reduzir a } \\
\text { dor e ajudar na } \\
\text { alteração do } \\
\text { comportamento em } \\
\text { crianças que sofrem } \\
\text { com tratamentos } \\
\text { oncológicos. Além de } \\
\text { fornecer recursos } \\
\text { educativos e } \\
\text { recomendações de } \\
\text { práticas melhores } \\
\text { para garantir o bem- } \\
\text { estar e a segurança } \\
\text { das pessoas } \\
\text { envolvidas neste tipo } \\
\text { de intervenção. }\end{array}$ & $\begin{array}{l}\text { O método etnográfico } \\
\text { foi selecionado para } \\
\text { investigar como um } \\
\text { cão de terapia pode } \\
\text { influenciar a cultura de } \\
\text { terapia pediátrica e } \\
\text { para explorar o } \\
\text { significado de cão na } \\
\text { prática de terapia } \\
\text { ocupacional } \\
\text { pediátrica. }\end{array}$ & $\begin{array}{l}\text { Estar perto de animais } \\
\text { tem uma ampla gama de } \\
\text { efeitos fisiológicos e } \\
\text { psicológicos positivos } \\
\text { para os humanos na } \\
\text { comunidade ou em } \\
\text { ambientes clínicos. }\end{array}$ \\
\hline $\begin{array}{c}\text { Maria Lindström } \\
\text { Nilsson, Eva-Lotta } \\
\text { Funkquist, Ann } \\
\text { Edner Gun Engval } \\
2019 \\
\text { (12) }\end{array}$ & $\begin{array}{c}50 \text { crianças de uma } \\
\text { enfermaria de cirurgia } \\
\text { pediátrica de um hospital } \\
\text { na Suécia. }\end{array}$ & $\begin{array}{l}\text { Avaliar experiências } \\
\text { e respostas de } \\
\text { crianças à terapia } \\
\text { assistida por animais } \\
\text { utilizando um cão de } \\
\text { terapia como } \\
\text { tratamento } \\
\text { complementar na } \\
\text { assistência hospitalar } \\
\text { pediátrica. }\end{array}$ & $\begin{array}{l}\text { O estudo foi realizado } \\
\text { por meio de métodos } \\
\text { mistos, utilizando } \\
\text { dados qualitativos e } \\
\text { quantitativos. }\end{array}$ & $\begin{array}{l}\text { Aumento do bem-estar } \\
\text { das crianças; pacientes } \\
\text { avaliaram a permanência } \\
\text { no hospital como melhor } \\
\text { após a terapia assistida } \\
\text { com cães. A grande } \\
\text { maioria das crianças } \\
\text { (93\%) avaliou a interação } \\
\text { com o cão como muito } \\
\text { boa. As crianças } \\
\text { descreveram sentimentos } \\
\text { de alegria, satisfação e } \\
\text { alívio da dor após a } \\
\text { terapia assistida por } \\
\text { animais. }\end{array}$ \\
\hline
\end{tabular}




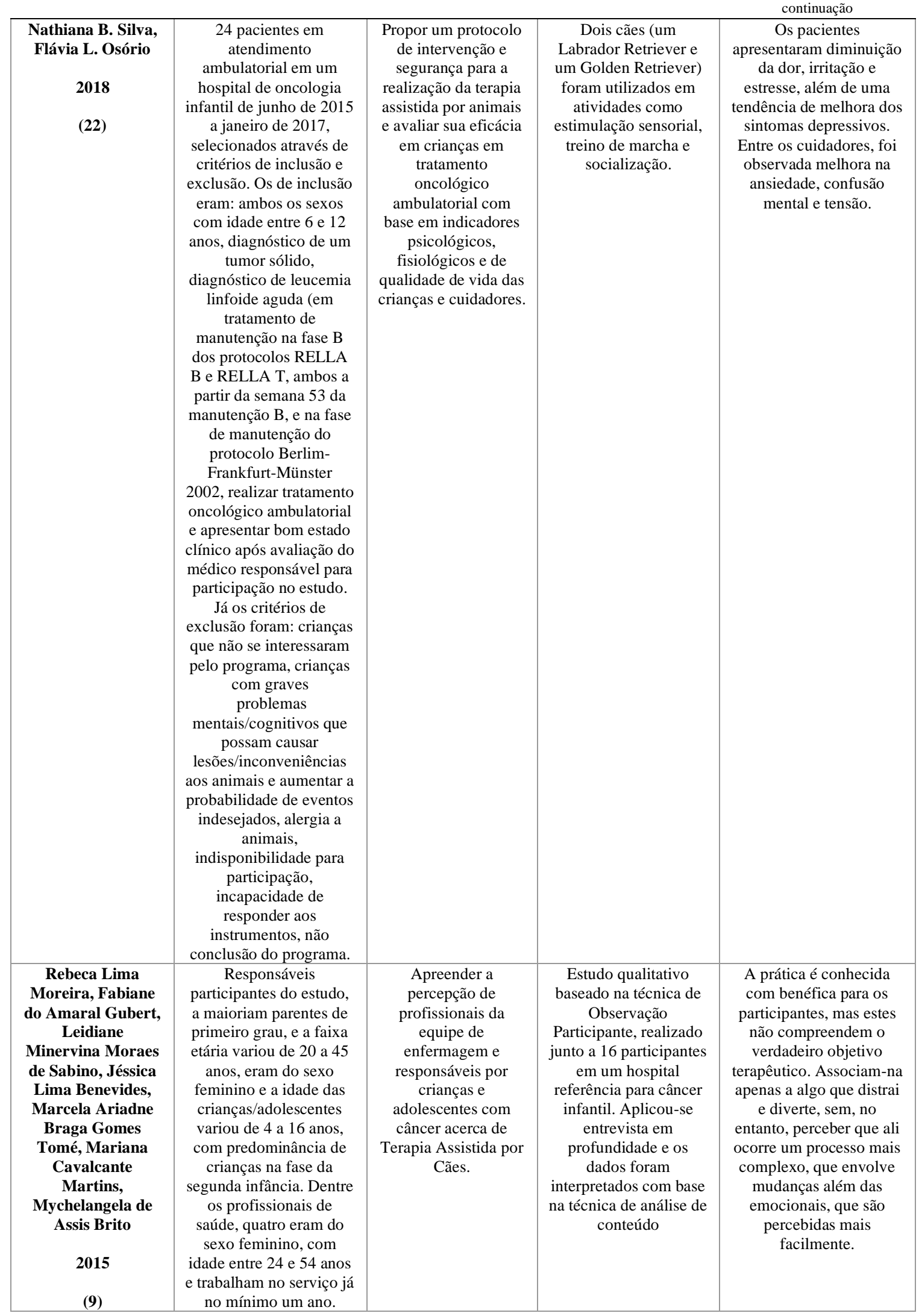

\section{Discussão}

A quimioterapia é o principal tratamento utilizado para pessoas diagnosticadas com câncer. Esse método de tratamento é sistêmico, atingindo de forma indiscriminada todas 
as células do organismo, principalmente células de rápida proliferação. Como não há especificidade celular, acarreta diversos efeitos colaterais, dentre os quais, náuseas, vômitos e queda de cabelo, levando à desgaste físico-emocional para o paciente $(7,8)$. Desta forma, tratamentos coadjuvantes, como a TAA com cães, podem ser utilizados para minimizar estes efeitos, além de colaborarem para um melhor enfrentamento da doença por parte dos doentes.

Moreira et al. (9) realizaram pesquisa em um hospital de referência para tratamento do câncer infanto-juvenil, avaliando a percepção de pais e enfermeiros quanto a utilização de TAA por cães na oncopediatria. Estes autores evidenciaram que TAA se mostrou um importante coadjuvante no tratamento de crianças com câncer, tornando os pacientes muito mais alegres e ativos; menos estressados e angustiados e melhorando a comunicação das crianças com os profissionais de saúde quando da presença do cão na sessão de quimioterapia. Os autores destacam que, segundo estudo realizado por Silveira et al. (10), ocorre maior liberação de endorfina e adrenalina quando se utilizam cães como auxiliares terapêuticos, minimizando-se assim os efeitos depressivos.

Ainda foi observada melhora na relação médico-paciente quando da presença de cães, o ambiente hospitalar se torna mais acolhedor e as crianças mais receptivas ao tratamento (9).

Em pesquisa realizada por Burr e Wittman (11), a escolha da raça dos cães se mostrou de suma importância para o desenvolvimento da criança na TAA, uma vez que o porte e o fenótipo do animal influenciam na conexão deste com o paciente. Um exemplo dessa situação é o caso de algumas crianças que ao observarem cães de grande porte sentem medo e acabam deixando de acariciá-lo. Assim, ver um animal considerado agradável e amigável motiva as crianças a brincar com eles, e isso contribui para promoção de mobilidade e agilidade nos movimentos, melhora na movimentação das mãos e um aumento no equilíbrio entre os pacientes. Esse é um dos principais benefícios relatados da TAA em pacientes oncológicos, já que é comum que eles fiquem tempos excessivos acamados devido à debilitação causada pelos tratamentos antineoplásicos aos quais são submetidos. Os autores destacam a Biofilia, já citada, como importante fator positivo de impacto, por gerar efeitos fisiológicos e psicológicos favoráveis aos humanos.

No estudo quanti-qualitativo realizado por Nilsson, Funkquist e Engval (12), em um hospital pediátrico da Suécia, houve melhora objetiva no bem-estar e nas experiências dessas crianças após sessão com os cães. Quando analisadas as vivências destas crianças com a doença e permanência hospitalar, antes e após a TAA, o estudo mostra que, inicialmente, aparecem vários pensamentos e sentimentos negativos, como dor causada pelo tratamento ou pela doença em si, medo de procedimentos e tristeza por conta do diagnóstico da doença. Após sessão de TAA, estas vivências modificam-se, com relatos de diminuição da dor quando o cachorro estava por perto, pela distração propiciada, fazendo com que estas enxerguem positivamente o momento.

A redução da dor, de acordo com Blalock e Smith (13) e Livnat et al. (14), ocorre devido ao papel da interação com animais de estimação na modulação da resposta psico-neuroimuno-endócrina. Assim, as emoções geram estímulos corticais que ativam tanto o sistema imune quanto o neuroendócrino, levando a liberação de neuro moduladores e hormônios que desencadeiam respostas bioquímicas ligadas à estímulos de base emocional. Desta forma, a interação dos cães com as crianças induz liberação de endorfina, a qual é responsável pela sensação de bem-estar, e de linfócitos, que aumentam a resposta imune (15).

Estudos (16-20) reforçam estes achados de estimulação psico-neuro-imuno-endócrina desencadeada pelo contato entre pessoas e animais. $\mathrm{O}$ ato de tocar e se comunicar, mesmo que visualmente, com o animal, acarreta maior liberação de ocitocina, serotonina e endorfinas, que combinadas contribuem diretamente para redução da ansiedade, medo e 
dor. Além disso, a interação promove diminuição do nível de cortisol basal no paciente, o que induz maior estado de relaxamento.

Importante ressaltar que pesquisas realizadas com métodos diferentes alcançaram resultados semelhantes. Os estudos de Chubak e Hawkes (21) e Silva e Osório (22) mostraram diminuição do cansaço, da dor e da ansiedade, porém os primeiros realizaram sessões de 20 minutos em um quarto privado do hospital, enquanto os segundos pesquisadores executaram três sessões de 30 minutos em grupo aberto. Esta última prática, TAA coletiva, está em ascensão, já que resulta em inúmeras vantagens advindas da troca de experiências e fortalecimento das relações interpessoais, que desenvolvem habilidades sociais e de comunicação nos pacientes.

Mesmo com tantos benefícios relatados acerca da utilização de TAA, há ainda certo receio por parte dos profissionais em utilizar esse tipo de tratamento, já que os pacientes diagnosticados com câncer ficam imunossuprimidos, o que culmina em uma maior facilidade de contágio por infecções oportunistas. Por isso, se faz necessário algumas medidas nos cães que realizam as visitas, tais como banho e higienização no animal previamente a ida ao hospital, realizações de exames para conferir se o cão não é portador de doenças transmissíveis, além de autorização pela Comissão de Infecção Hospitalar. Somado a isso, deve-se sempre considerar a vontade do paciente em receber a visita do cão para uma assistência ao tratamento $(23,24)$.

Tendo em vista estas considerações, Gillespie e Neu (25) realizaram TAA por ambiente virtual, o que possibilitou a interação da criança com o cão ou gato. As crianças escolhem seus animais, passam por entrevistas e se comunicam com estes por meio da troca de cartas acompanhadas de imagens, a fim de oferecer um maior conforto e acolhimento para os pacientes. Após o processo é perceptível que os enfermos apresentavam os benefícios comuns à TAA realizada com visitas, além da facilidade em expressar seus sentimentos e angústias relacionadas ao tratamento por meio da escrita. Este artigo ainda ressalta que dos 15 participantes, 14 ganharam um cão ou gato após o tratamento, uma vez que foi criado um forte vínculo de amizade.

Os artigos demonstram que mesmo a TAA sendo um complemento promissor no tratamento das crianças, essa prática não se encontra muito difundida nos hospitais, principalmente no Brasil. Essa falta de adesão ao tratamento ocorre por falta de conhecimento da prática, de todos os benefícios que esta promove, além de ainda acreditarem que os animais possuem função meramente interativa para os pacientes.

\section{Conclusão}

A partir dessa revisão integrativa, abordando o tema Terapia Assistida por Cães na Pediatria Oncológica, é notório que esse tipo de tratamento acarreta inúmeros benefícios para o paciente, como foi destacado - melhora na socialização, ânimo, bem-estar e atividade física. Além disso também ficou claro que os profissionais da saúde conseguem realizar um melhor manejo e adesão da criança perante o tratamento.

Sabe-se que ainda há carência de estudos nessa área, entretanto é perceptível os resultados promissores que os cães podem trazer para auxiliar na terapia convencional, amenizando efeitos depressivos e deixando o hospital um ambiente mais descontraído. Cabe a ressalva de que os cães não são escolhidos aleatoriamente, e devem passar por procedimentos controlados por veterinários para poderem realizar as visitas, a fim de não causarem danos aos pacientes. 


\section{Referências}

1. Zanatta AA, Santos Jr Robiran J, Perini CC, Fischer ML. Biofilia: produção de vida ativa em cuidados paliativos. Saúde debate. 2019; 43(122): 949-965.

2. Teixeira IS. A terapia assistida por animais como uma forma de associação: um estudo antropológico sobre a relação humano-animais na promoção da saúde humana, no Brasil [Tese de Doutorado] Porto Alegre: Programa de Pós-graduação em Antropologia Social, Universidade Federal do Rio Grande do Sul. 2015.

3. Ribeiro AFA. Cães domesticados e os benefícios da interação. Revista Brasileira de Direito Animal. $2011 ; 8: 249-262$.

4. Da Silva NB; Raniero EP; Alvarez CDL. Benefícios da terapia assistida por animais no desenvolvimento motor de crianças com Síndrome de Down. Saúde, Batatais. 2014; 2(1): 67-82.

5. McCune S; Kruger KA; Griffin JA, Esposito L, Freund LS, Hurley KJ, Bures R. Evolution of research into the mutual benefits of human-animal interaction, Animal Frontiers. 2014; 4(3): 49-58.

6. Moher D; Liberati A; Tetzlaff J; Altman DG. The PRISMA Group. Preferred reporting items for systematic reviews and meta-analyses: The PRISMA statement. PLoS Med 2009; 6(7): e1000097.

7. Bonassa EMA, Santana TR. Enfermagem em terapêutica oncológica. 3 ed. São Paulo: Atheneu, 2005.

8. Soares L.C; Burille A.; Antonacci M.H; Santana M.G; Schwartz E. A quimioterapia e seus efeitos adversos: relato de clientes oncológicos. Cogitare Enferm. 2009; 4(14): 714-9.

9. Moreira RL, Gubert FA, Sabino LMM, Benevides JL, Tomé MABG, Martins MC et al. Terapia assistida com cães em pediatria oncológica: percepção de pais e enfermeiros. Rev. Bras. Enferm. 2016; 69(6): 1188-1194.

10. Silveira IR, Santos NC, Linhares DR. Protocolo do Programa de Assistência Auxiliada por Animais no Hospital Universitário. Rev. esc. enferm. USP. 2011; 45(1): 283-288.

11. Burr M, Wittman P. The influence of a therapy dog on a pediatric therapy organization: A mini ethnography. Complement Ther Clin Pract. 2020; 38:101083.

12. Lindström NM, Funkquist EL, Edner A, Engvall G. Children report positive experiences of animalassisted therapy in paediatric hospital care. Acta Paediatr. 2020; 109(5):1049-1056.

13. Blalock JE, Smith EM. Human leukocyte interferon (HuIFN-alpha): potent endorphin-like opioid activity. Biochem Biophys Res Commun. 1981; 101(2): 472-8.

14. Livnat S, Felten SY, Carlson SL, Bellinger DL, Felten DL. Involvement of peripheral and central catecholamine systems in neural-immune interactions. J Neuroimmunol. 1985; 10(1): 5-30.

15. Braun C, Stangler T, Narveson J, Pettingell S. Animal-assisted therapy as a pain relief intervention for children. Complementary Therapies in Clinical Practice. 2009; 15(2): 105-109.

16. Golfeto JH, Veiga MH, Souza L de, Barbeira CB dos S. Propriedades psicométricas do inventário da depressão infantil (CDI) aplicado em uma amostra de escolares de Ribeirão Preto. Revista de Psiquiatria Clínica. 2002; 29(2): 66-70.

17. Terry PC, Lane AM, Fogarty GJ. Construct validity of the POMS-A for use with adults. Psychology of Sport and Exercise. 2003; 4: 125-39.

18. Trembath F. Animal-assisted intervention for people with cancer. Habri Central. 2015; (17): 1-5.

19. Albuquerque NS; Ciari MB. Cães e seres humanos: uma relação forte, complexa, duradoura e vantajosa. In: Chelini MOM, Otta E, editors. Terapia Assistida por Animais. São Paulo: Editora Manole; 2016. p. 18-20.

20. Hicks CL; Von Baeyer CL; Spafford PA; Van Korlaar I; Goodenough B. The Faces Pain ScaleRevised: toward a common metric in pediatric pain measurement. Pain. 2001; 93(2): 173-183.

21. Chubak J, Hawkes R, Dudzik C, Foose-foster J, Eaton L, Johnson R, Macpherson C. Pilot study of therapy dog visits for inpatient youth with cancer. J Pediatr Oncol Nurs. 2017; 34(5): 331-341.

22. Silva NB, Osório FL. Impact of an animal-assisted therapy programme on physiological and psychosocial variables of paediatric oncology patients. PLoS One. 2018; 13(4): e0194731.

23. Schmitz A, Beermann M, MacKenzie C.R. et al. Animal-assisted therapy at a University Centre for Palliative Medicine - a qualitative content analysis of patient records. BMC Palliat Care. 2017; 16(1): 50.

24. Odendaal JS. Animal-assisted therapy - magic or medicine? J Psychosom Res. 2000; 49(4): 275-8.

25. Gillespie AI, Neu M. Youth and Pet Survivors: Exploring the Experiences of Pediatric Oncology and Bone Marrow Transplant Patients in a Virtual Animal-Assisted Therapy Pen Pal Program. J Pediatr Oncol Nurs. 2020; 37(6):368-376. 